\title{
Successful shrinkage of anterior communicating artery aneurysm after ACA-ACA bypass with interposed occipital artery graft in pediatric moyamoya disease: illustrative case
}

\author{
Kota Nakajima, MD, Takeshi Funaki, MD, PhD, Masakazu Okawa, MD, PhD, Kazumichi Yoshida, MD, PhD, and \\ Susumu Miyamoto, MD, PhD
}

Department of Neurosurgery, Kyoto University Graduate School of Medicine, Kyoto, Japan

BACKGROUND Selecting therapeutic options for moyamoya disease (MMD)-associated anterior communicating artery (ACoA) aneurysm, a rare pathology in children, is challenging because its natural course remains unclear.

OBSERVATIONS A 4-year-old boy exhibiting transient ischemic attacks was diagnosed with unilateral MMD accompanied by an unruptured ACOA aneurysm. Although superficial temporal artery to middle cerebral artery anastomosis eliminated his symptoms, the aneurysm continued to grow after surgery. Since a previous craniotomy and narrow endovascular access at the ACoA precluded both aneurysmal clipping and coil embolization, the patient underwent a surgical anastomosis incorporating an occipital artery graft between the bilateral cortical anterior cerebral arteries (ACAs). This was intended to augment blood flow in the ipsilateral ACA territory and to reduce the hemodynamic burden on the ACoA complex. The postoperative course was uneventful, and radiological images obtained 12 months after surgery revealed good patency of the bypass and marked shrinkage of the aneurysm in spite of the intact contralateral internal carotid artery.

LESSONS Various clinical scenarios should be assessed carefully with regard to this pathology. Bypass surgery aimed at reducing flow to the aneurysm might be an alternative therapeutic option when neither coiling nor clipping is feasible.

https://thejns.org/doi/abs/10.3171/CASE21460

KEYWORDS pediatric moyamoya disease; anterior communicating artery aneurysm; ACA-ACA anastomosis; case report

Anterior communicating artery $(\mathrm{ACOA})$ aneurysm is a possible complication of moyamoya disease (MMD). It is characterized by chronic progressive stenosis of the terminal portion of the internal carotid artery (ICA). ${ }^{1}$ ACoA aneurysm occurs specifically in unilateral MMD (MMD with unilateral ICA involvement); it is hypothesized that the hemodynamic burden on the ACOA complex is increased by the collateral flow from the contralateral ICA. ${ }^{2-4}$ ACOA aneurysm is occasionally associated with adult MMD. ${ }^{2-8}$ Reports of $A C O A$ aneurysm associated with pediatric MMD are rare, however. Aneurysmal coil embolization and clipping, typical therapeutic options for MMD-associated aneurysms, ${ }^{3-6,8}$ are not always feasible in young children, and an optimal treatment strategy remains unresolved for this population. Furthermore, treatment decisions are sometimes difficult because patients with unilateral moyamoya disease often exhibit contralateral progression, which might lead to spontaneous shrinkage of an $\mathrm{ACOA}$ aneurysm later on. ${ }^{9}$

We describe a rare case of a young child with unilateral MMD developing an ACoA aneurysm after superficial temporal artery-tomiddle cerebral artery (STA-MCA) anastomosis. The patient was successfully treated with a unique surgical bypass, intended to reduce the hemodynamic burden on the ACOA complex.

\section{Illustrative Case}

\section{Clinical Presentation}

A 4-year-old boy experienced transient weakness on the left side of the body when crying and was referred to our department.

ABBREVIATIONS ACA = anterior cerebral artery; $\mathrm{ACOA}=$ anterior communicating artery; $\mathrm{CT}=$ computed tomography; ICA $=$ internal carotid artery; $\mathrm{MCA}=$ middle cerebral artery; $\mathrm{MMD}$ = moyamoya disease; $\mathrm{OA}=$ occipital artery; $\mathrm{SPECT}$ = single-photon emission computed tomography; STA = superficial temporal artery. INCLUDE WHEN CITING Published October 25, 2021; DOI: 10.3171/CASE21460.

SUBMITTED August 11, 2021. ACCEPTED August 25, 2021.

(c) 2021 The authors, CC BY-NC-ND 4.0 (http://creativecommons.org/licenses/by-nc-nd/4.0/). 


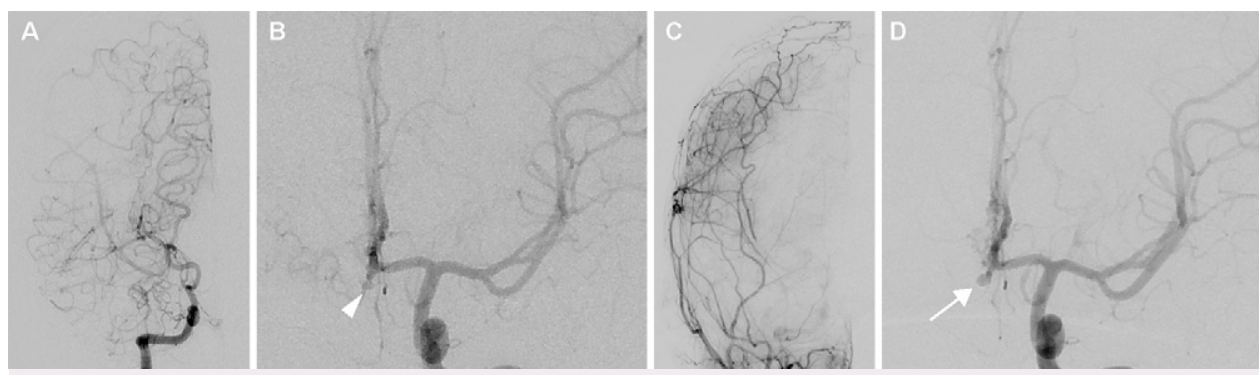

FIG. 1. Serial angiographic images. A: Anterior-posterior view of right internal carotid angiography on first admission. B: Anterior-posterior view of left internal carotid angiography on first admission showing a small ACOA aneurysm (arrowhead). C: Anterior-posterior view of right external carotid angiography obtained 3 months after STA-MCA anastomosis. D: Anterior-posterior view of left internal carotid angiography obtained 16 months after STA-MCA anastomosis. Note the apparent enlargement of the ACoA aneurysm (arrow).

Angiography on admission revealed severe stenosis in the terminal portion of the right ICA with development of a collateral network (Fig. 1A), and the patient was diagnosed with unilateral MMD. The terminal portion of the left ICA was intact, and a very small aneurysm was observed at the ACoA complex (Fig. 1B). The patient underwent STA-MCA anastomosis of the right hemisphere, and his transient ischemic attacks ceased after surgery. Angiography performed 3 months after surgery revealed good patency of the STA-MCA anastomosis (Fig. 1C). A wait-and-see approach was applied to the ACoA aneurysm, and the patient underwent biannual magnetic resonance imaging scans. However, the scans eventually suggested gradual enlargement of the ACoA aneurysm. Angiography performed 16 months after surgery revealed apparent enlargement of the aneurysm (Fig. 1D). Three-dimensional rotational angiography revealed a saccular aneurysm, $3.9 \mathrm{~mm}$ in diameter, located near the right A1-A2 junction (Fig. 2). Single-photon emission computed tomography (SPECT) revealed a moderate decrease in cerebral blood flow in the right anterior cerebral artery (ACA) territory.

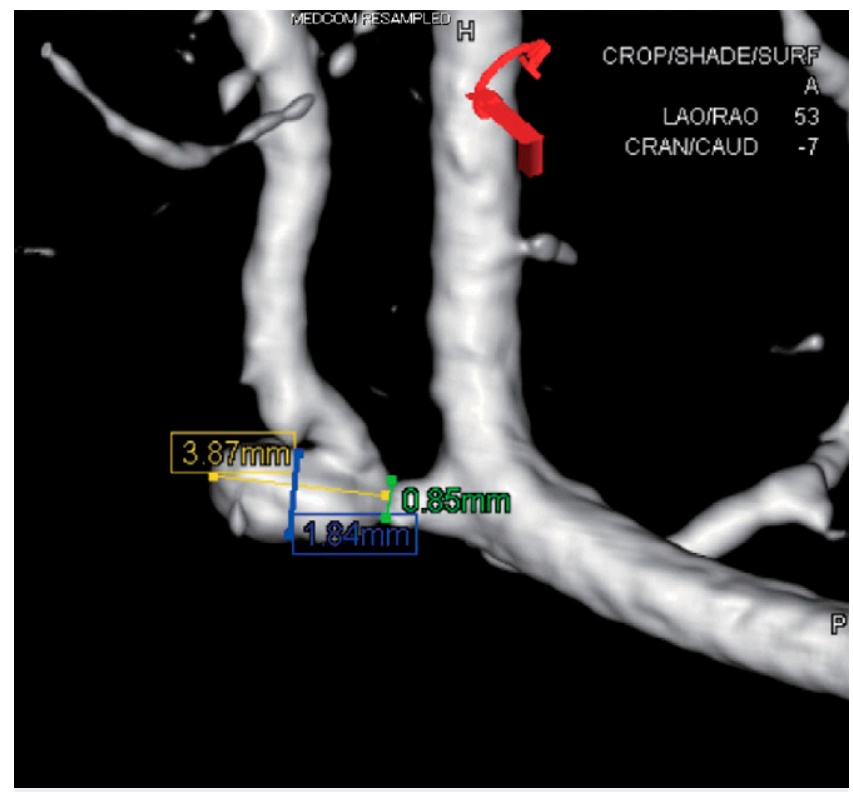

FIG. 2. Three-dimensional rotational angiography from left common carotid arterial injection.

\section{Therapeutic Assessment}

As a therapeutic option for preventing rupture of the aneurysm, aneurysmal coil embolization and direct clipping of the aneurysm were first considered. However, the former option was abandoned because the ACoA, the only endovascular access to the aneurysm, was less than $1 \mathrm{~mm}$ in diameter, and the microcatheter was at risk of becoming wedged in the ACoA. Moreover, clipping via the right pterional approach was also unfeasible because the patient had already undergone right STA-MCA anastomosis. Natural shrinkage of the aneurysm could potentially occur if the contralateral ICA gradually became occluded in the future; however, the parents no longer wished to take a wait-and-see approach because of the risk of fatal rupture.

Accordingly, an alternative surgical strategy intended to reduce the hemodynamic burden on the ACoA complex was considered. As the aneurysmal growth was reasonably attributable to increased flow demand in the right $A C A$, we chose bypass surgery targeting the mild ischemia in the right ACA territory; specifically, we chose ACA-to-ACA (ACA-ACA) anastomosis incorporating a left occipital artery (OA) graft (Fig. 3).

\section{Surgical Procedure}

Preoperative computed tomography (CT) angiography, through which bilateral cortical ACAs were exposed on the surface of the

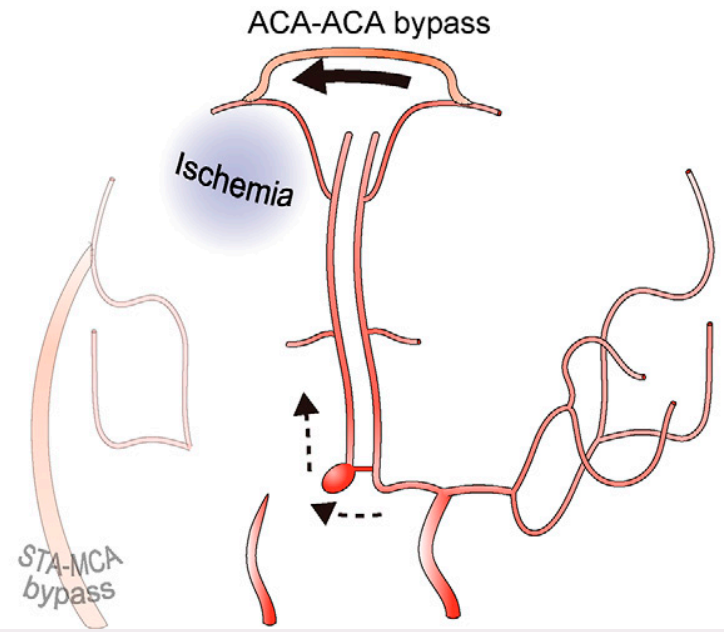

FIG. 3. Schematic drawing of ACA-ACA anastomosis with interposed OA graft adopted for the patient. 

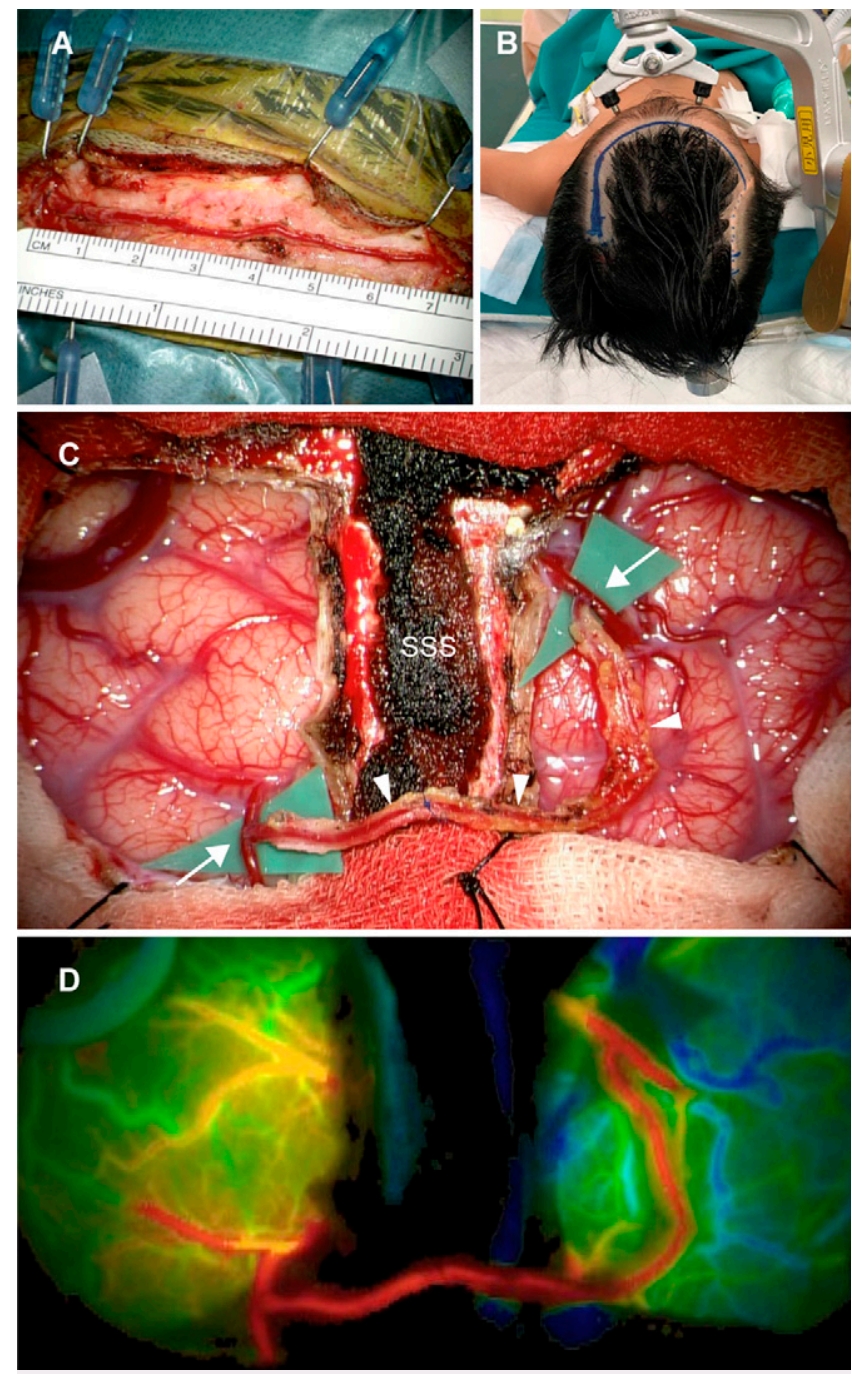

FIG. 4. Intraoperative findings. A: Left OA harvested as a free graft from a linear skin incision. B: Patient position and skin incision for ACA-ACA anastomosis. The skin incision line shares part of that made for the previous right STA-MCA anastomosis. C: Photo obtained after completion of ACA-ACA anastomosis. Arrows indicate the anastomosis site; arrowhead, OA graft. SSS = superior sagittal sinus. D: Indocyanine green video angiography obtained after anastomosis.

brain, was performed to simulate the craniotomy site. At the beginning of the surgery, the patient was placed in the lateral position, and the left $O A$ was harvested as a $7-\mathrm{cm}$ free graft through a linear skin incision (Fig. 4A). He was subsequently repositioned from a lateral to a spine position. A horseshoe-shaped skin incision, allowing a further anterior or left-side craniotomy, was made behind the hairline of the forehead. Particular attention was paid to preserve the parietal branch of the left STA (Fig. 4B). A midline craniotomy centered at the Bregma was performed. After the dura was opened, a cortical branch of the ACA was identified in each hemisphere, and these were prepared as anastomosis sites. A cut end of the OA graft was first anastomosed to the left ACA by end-to-side anastomosis; the other end of the graft was anastomosed to the right $\mathrm{ACA}$ in the same manner (Fig. $4 \mathrm{C}$ ). Before the last suture was tied, the temporary clip was released for an instant to flush the air and confirm the blood flow from the contralateral anastomosis site. After completion of the anastomosis, indocyanine green video angiography revealed good patency of the bypass with left-to-right flow direction through the graft (Fig. 4D). Surgical procedures are also shown in Video 1.

VIDEO 1. Clip showing ACA-ACA anastomosis with interposed $\mathrm{OA}$ graft. Click here to view.

\section{Outcome}

The postoperative course was uneventful, and the patient was discharged shortly after surgery without surgical complication. Angiography performed 5 months after surgery revealed good patency of the ACA-ACA bypass with evidence of aneurysmal shrinkage (Fig. 5 left). SPECT performed at the same admission revealed improved blood flow in the right ACA territory. CT angiography performed 12 months after surgery revealed further marked shrinkage of the aneurysm, while the left ICA remained intact (Fig. 5 right). As of this writing, the aneurysm has remained free from regrowth for 24 months.

\section{Discussion}

\section{Observations}

The present case report includes two remarkable observations: first, an ACOA aneurysm was associated with pediatric MMD; and second, a unique therapeutic design facilitating flow reduction at the ACoA complex resulted in successful shrinkage of the aneurysm.

Reports of ACoA aneurysm associated with pediatric MMD are rare as of this writing. Noureldine et al. reported the case of a 14year-old girl exhibiting subarachnoid hemorrhage due to a ruptured ACA aneurysm associated with MMD. ${ }^{10}$ The nature of the aneurysm, however, seemed to differ from that of the present case because the aneurysm was located at the A1 portion, and a dissecting aneurysm was suspected. Within their case series of MMDassociated aneurysms, Liu et al. reported a 9-year-old boy with an

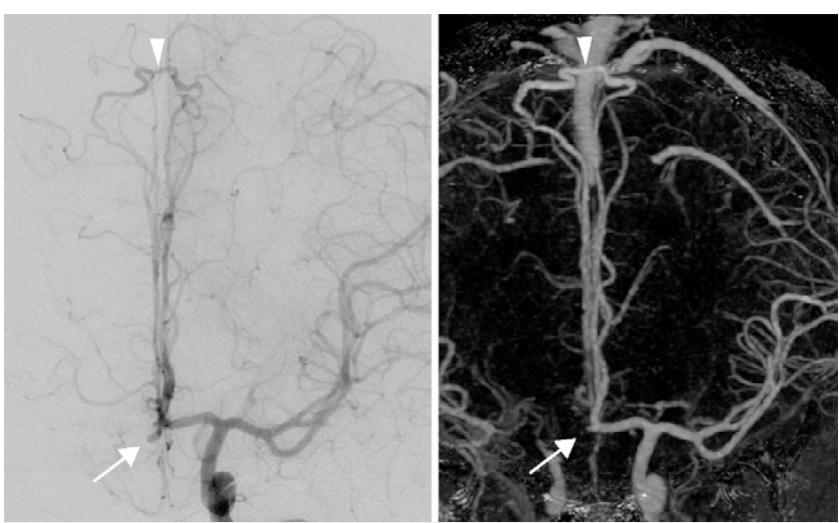

FIG. 5. Serial radiographic images after ACA-ACA anastomosis. Left: Anterior-posterior view of left internal carotid angiography obtained 5 months after ACA-ACA anastomosis. Right: Anterior-posterior view of CT angiography obtained 12 months after ACA-ACA anastomosis. Note the remarkable shrinkage of the $\mathrm{ACOA}$ aneurysm. Arrows indicate the ACoA aneurysm; arrowhead, patent ACA-ACA anastomosis. 
unruptured ACoA aneurysm. ${ }^{11}$ Although little information is available regarding this patient, he underwent conservative treatment, and the aneurysm remained present.

The favorable change in the aneurysm observed in the present case might be explained by a hypothesis regarding aneurysmal formation in MMD. Kim et al. have hypothesized that progressive occlusion of the ICA jeopardizes hemodynamics in other arteries, causing compensatory dilation and increased blood flow through collateral vessels, which often lead to the formation and rupture of aneurysms. ${ }^{2}$ This hypothesis suggests the potential benefit of the flow-reduction strategy using bypass to counter aneurysmal growth. This speculation is in line with several reports showing that aneurysms located on the basilar or choroidal artery can shrink after bypass surgery for stenoocclusive diseases in the anterior circulation. ${ }^{12,13}$ On the other hand, an aneurysm in a young child is an unusual occurrence in itself, and a direct causal relationship between MMD and aneurysm formation has not been proved in the present case.

The bypass surgery illustrated here could offer an alternative therapeutic option for ACoA aneurysms associated with pediatric $M M D$, where neither clipping nor aneurysmal coil embolization is feasible. Even in adult cases, clipping of MMD-associated ACoA aneurysms is challenging because of the relatively high risk of ischemic complication. ${ }^{14}$ Although aneurysmal coil embolization for MMD-associated ACOA aneurysms shows excellent results overall, ${ }^{3,5}$ the procedure might not always be feasible in pediatric patients because endovascular access is limited. We did not adopt A3-A3 side-to-side anastomosis ${ }^{15}$ in the present case because our procedures seemed less invasive in terms of brain retraction.

Various clinical scenarios should be considered with care before beginning treatment of MMD-associated ACoA aneurysms, especially in pediatric patients, as contralateral disease progression occurs in about half of all patients with unilateral MMD. ${ }^{9}$ In this respect, our therapeutic design might be significant in providing further surgical options, including contralateral STA-MCA anastomosis. The natural clinical course of MMD-associated ACoA aneurysm remains unknown. While some researchers have stressed the devastating nature of MMD-associated aneurym, ${ }^{16,17}$ others have suggested that such aneurysms can spontaneously disappear with the progression of MMD. ${ }^{18,19}$ Spontaneous disappearance of $A C O A$ aneurysm can occur in unilateral MMD if the contralateral ICA gradually becomes occluded. Our therapeutic design might be acceptable even in this scenario; however, long-term follow-up should be required in the present case.

\section{Lessons}

Various treatment options should be carefully assessed for MMDassociated $\mathrm{ACOA}$ aneurysms in pediatric patients because their natural course has not been sufficiently revealed. ACA-ACA bypass with interposed $O A$ graft, which is intended to reduce flow at the ACoA complex, might be an alternative treatment option for a growing $A C D A$ aneurysm associated with pediatric MMD, where neither coil embolization nor clipping is feasible.

\section{References}

1. Research Committee on the Pathology and Treatment of Spontaneous Occlusion of the Circle of Willis. Guidelines for diagnosis and treatment of moyamoya disease (spontaneous occlusion of the circle of Willis). Neurol Med Chir (Tokyo). 2012;52(5): 245-266.
2. Kim JH, Kwon TH, Kim JH, Chong K, Yoon W. Intracranial aneurysms in adult moyamoya disease. World Neurosurg. 2018;109:e175-e182.

3. Yanagida T, Ichikawa T, Suzuki K, Watabe Y. A case of ruptured anterior communicating artery aneurysm associated with unilateral moyamoya disease treated by endovascular coil embolization. Article in Japanese. Surg Cereb Stroke. 2015;43:223-227.

4. Kasamo S, Asakura T, Yamamoto Y, Kobayashi E. Unilateral moyamoya disease associated with multiple aneurysms. A case report and review of the literature. Neurol Med Chir (Tokyo). 1984;24(1): 30-34.

5. Yu JL, Wang HL, Xu K, Li Y, Luo Q. Endovascular treatment of intracranial aneurysms associated with moyamoya disease or moyamoya syndrome. Interv Neuroradiol. 2010;16(3):240-248.

6. Iwama T, Todaka T, Hashimoto N. Direct surgery for major artery aneurysm associated with moyamoya disease. Clin Neurol Neurosurg. 1997;99(Suppl 2):S191-S193.

7. Kawaguchi S, Sakaki T, Morimoto T, Kakizaki T, Kamada K. Characteristics of intracranial aneurysms associated with moyamoya disease. A review of 111 cases. Acta Neurochir (Wien). 1996;138(11): 1287-1294.

8. Yaşargil MG, Smith RD. Association of middle cerebral artery anomalies with saccular aneurysms and moyamoya disease. Surg Neurol. 1976;6(1):39-43.

9. Park EK, Lee YH, Shim KW, Choi JU, Kim DS. Natural history and progression factors of unilateral moyamoya disease in pediatric patients. Childs Nerv Syst. 2011;27(8):1281-1287.

10. Noureldine MHA, Saikali I, Nassif A, et al. Pediatric moyamoya presenting as a subarachnoid hemorrhage from a ruptured anterior cerebral artery aneurysm. World Neurosurg. 2020;134: 123-127.

11. Liu P, Lv XL, Liu AH, et al. Intracranial aneurysms associated with moyamoya disease in children: clinical features and long-term surgical outcome. World Neurosurg. 2016;94:513-520.

12. Indo M, Oya S, Matsui T. Ruptured basilar tip aneurysm in a patient with bilateral internal carotid artery occlusion successfully treated with bilateral superficial temporal artery-middle cerebral artery anastomoses: case report. World Neurosurg. 2016;86:512. e5-512.e8.

13. Kuroda S, Houkin K, Kamiyama $H$, Abe $H$. Effects of surgical revascularization on peripheral artery aneurysms in moyamoya disease: report of three cases. Neurosurgery. 2001;49(2):463-468.

14. Yeon JY, Kim JS, Hong SC. Incidental major artery aneurysms in patients with non-hemorrhagic moyamoya disease. Acta Neurochir (Wien). 2011;153(6):1263-1270.

15. Mabuchi S, Kamiyama H, Kobayashi N, Abe H. A3-A3 side-to-side anastomosis in the anterior communicating artery aneurysm surgery: report of four cases. Surg Neurol. 1995;44(2): 122-127.

16. Kim S, Jang CK, Park EK, et al. Clinical features and outcomes of intracranial aneurysm associated with moyamoya disease. J Clin Neurol. 2020;16(4):624-632.

17. Ni W, Jiang $H, X u B$, et al. Treatment of aneurysms in patients with moyamoya disease: a 10-year single-center experience. $J$ Neurosurg. 2018;128(6):1813-1822.

18. Zhang L, Xu K, Zhang Y, Wang X, Yu J. Treatment strategies for aneurysms associated with moyamoya disease. Int J Med Sci. 2015;12(3):234-242.

19. Satoh T, Yamamoto Y, Asari S, Sakurai M, Suzuki K. Disappearance and development of cerebral aneurysms in moyamoya disease. Case report. J Neurosurg. 1983;58(6):949-953.

\section{Disclosures}

The authors report no conflict of interest concerning the materials or methods used in this study or the findings specified in this paper. 


\section{Author Contributions}

Conception and design: Funaki, Nakajima, Miyamoto. Acquisition of data: Funaki, Nakajima. Analysis and interpretation of data: all authors. Drafting the article: Funaki, Nakajima, Okawa. Critically revising the article: Funaki, Nakajima, Yoshida, Miyamoto. Reviewed submitted version of manuscript: all authors. Approved the final version of the manuscript on behalf of all authors: Funaki. Administrative/technical/ material support: Miyamoto. Study supervision: Miyamoto.

\section{Supplemental Information}

Video

Video 1. https://vimeo.com/592761248.

\section{Correspondence}

Takeshi Funaki: Graduate School of Medicine, Kyoto University, Kyoto, Japan. tfunaki@kuhp.kyoto-u.ac.jp. 